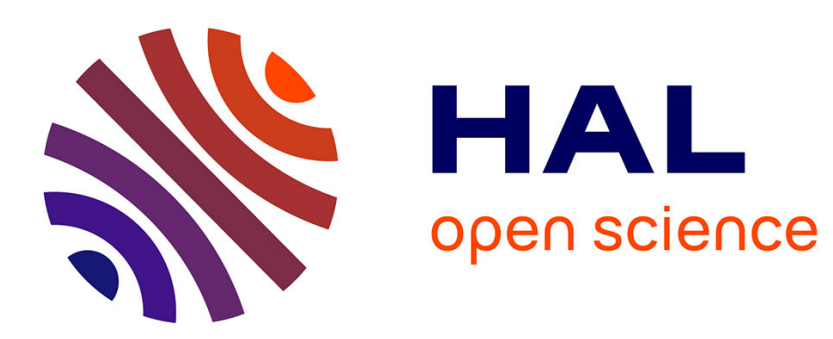

\title{
Observation des défauts dans les semiconducteurs par microscopie à balayage en cathodoluminescence
}

\author{
C. Schiller, D. Bois
}

\section{To cite this version:}

C. Schiller, D. Bois. Observation des défauts dans les semiconducteurs par microscopie à balayage en cathodoluminescence. Revue de Physique Appliquée, 1974, 9 (2), pp.361-371. 10.1051/rphysap:0197400902036100 . jpa-00243788

\section{HAL Id: jpa-00243788 https://hal.science/jpa-00243788}

Submitted on 1 Jan 1974

HAL is a multi-disciplinary open access archive for the deposit and dissemination of scientific research documents, whether they are published or not. The documents may come from teaching and research institutions in France or abroad, or from public or private research centers.
L'archive ouverte pluridisciplinaire HAL, est destinée au dépôt et à la diffusion de documents scientifiques de niveau recherche, publiés ou non, émanant des établissements d'enseignement et de recherche français ou étrangers, des laboratoires publics ou privés. 


\title{
OBSERVATION DES DÉFAUTS DANS LES SEMICONDUCTEURS PAR MICROSCOPIE A BALAYAGE EN CATHODOLUMINESCENCE
}

\author{
C. SCHILLER et D. BOIS
}

Laboratoire d'Electronique et de Physique Appliquée, 3, avenue Descartes, 94450 Limeil Brévannes, France

\begin{abstract}
Résumé. - Il a précédemment été montré que le microscope électronique à balayage permettait la visualisation de défauts tels que dislocations et précipitations dans les semiconducteurs composés à grand gap.

Nous étudierons ici les conditions de formation de ces images du point de vue de la résolution et de la profondeur concernée ainsi que de la nature des inhomogénéités pouvant conduire à un contraste d'image. On montre que deux cas doivent être considérés suivant le dopage du matériau et le taux d'injection. L'influence de la diffusion des porteurs minoritaires, des recombinaisons en surface et de l'auto-absorption est également envisagée.

On décrit des exemples d'observation des dislocations et des précipités dans les monocristaux de GaAs dopés, massifs ou épitaxiques, et dans des composés pseudo-binaires. En faisant varier le taux d'injection, ces mêmes défauts peuvent être observés dans les matériaux peu dopés ou semiisolants.

On étudiera également la variation de l'intensité de cathodoluminescence des couches $\mathrm{n}^{+} / \mathrm{n}$ sur substrat $\mathrm{n}^{+}$. L'analyse de ces résultats fait ressortiir l'influence de la diffusion des défauts autour des dislocations dans les matériaux dopés, ainsi que des phénomènes semblables d'accumulation de centres non radiatifs dans le cas des matériaux purs. De même, des effets de diffusion du cuivre et de propagation de défauts non radiatifs apparaissent au voisinage des interfaces substrat/épitaxie.
\end{abstract}

\begin{abstract}
It has been previously shown that the visualisation of defects such as dislocations and precipitates in compound semiconductors was possible in a scanning electron microscope. We will study here conditions of image formation from the point of view of resolution and depth involved as well as the nature of inhomogeneities creating an image contrast. We show that two cases have to be considered related to the doping level of materials and to the injection range. The influence of minority carriers diffusion, surface recombination and self absorption has also been considered.

We describe examples of observations on dislocations and precipitates in doped monocrystalline GaAs, bulk and epitaxial, and in pseudobinary compounds. By an increase of the injection rate, similar defects are observed in low doped and semi insulating materials. We also study the variations of cathodoluminescence intensities of $n^{+} / n$ on $n^{+}$substrates. Analysis of results emphasizes the influence of defects diffusion around dislocations in doped materials with similar effects of non radiative centers accumulation in pure materials. In the same way, diffusion effects of copper and propagation of non radiative defects appear in the vicinity of interfaces substrate/epitaxial layer.
\end{abstract}

1. Introduction. - On sait que la plupart des corps solides soumis à un bombardement électronique émettent de la lumière. Plusieurs mécanismes de retour à l'équilibre des électrons du solide excités par le choc des électrons du faisceau primaire peuvent provoquer cette émission de cathodoluminescence. Dans les semiconducteurs, deux processus prédominent : les transitions bande de conduction-bande de valence ou en niveaux voisins de ces bandes et les transitions mettant en jeu des niveaux associés à des éléments et situés loin des bandes permises du solide parfait. Le premier type de transition est prépondérant dans les semiconducteurs à structure de bande directe, alors que les deux types se mélangent dans les autres semiconducteurs.

De nombreuses études spectrales, en particulier à basse température de la cathodoluminescence sont rapportées dans la littérature (pour GaAs par exemple [1], [2], [3], [4], [5]) de sorte que pour chaque corps, les spectres d'émission sont assez bien connus. Il apparaît que ces spectres dépendent beaucoup, pour un matériau donné des caractéristiques des échantillons, par exemple de leur dopage, du type et de la densité des impuretés et des défauts cristallins. Par conséquent, la cathodoluminescence constitue un bon moyen de mise en évidence de ces défauts. 
De plus, en utilisant une microsonde électronique, ou un microscope à balayage électronique il devient possible de réaliser des études ponctuelles et de faire des images de surface des divers matériaux. Les premières mesures dans ce domaine ont été réalisées par Pinard [6] sur des halogénures alcalins.

De nombreuses analyses de ce type ont ensuite été réalisées sur des semiconducteùrs, principalement sur GaAs [7], [8], [9], [10], [11], [12], [13], [14] ; elles ont montré que l'on pouvait observer par ce moyen des dislocations, des précipités et des hétérogénéités de dopage dans des cristaux de GaAs très dopés. Cette méthode constitue donc un mode d'utilisation du MEB qui complète les analyses classiques par émission électronique secondaire et rétrodiffusée qui sont surtout sensibles à des phénomènes de surface et à la morphologie des échantillons. Par contre la cathodoluminescence s'applique à l'étude des défauts de volume dans les matériaux, de même que les études en courant induit, mais celles-ci nécessitent la réalisation d'une structure soit à l'aide d'une barrière métal-semiconducteur, soit par diffusion, et de telles structures ne sont pas réalisables sur tous les matériaux. Dans une précédente étude, nous avons montré les corrélations qui existent entre les images de courant induit et celles de cathodoluminescence dans des diodes électroluminescentes en GaAs [15]. Nous avons également utilisé ce type de formation d'image pour la caractérisation de GaAs [16] et nous l'avons appliqué pour étudier la propagation des défauts dans les couches épitaxiques graduelles de composés pseudo-binaires tels que GaAsP [17] et GaInAs [18].

Dans ce qui suit, nous préciserons les conditions de formation des images de cathodoluminescence du point de vue de la résolution, de la profondeur concernée, de l'influence de la surface, de l'auto-absorption, de la longueur de diffusion, de la géométrie de l'échantillon et des paramètres du faisceau (tension d'accélération et débit).

Nous discuterons aussi l'origine des contrastes observés en fonction du dopage des échantillons et du taux d'injection. Du point de vue expérimental nous avons été conduits à faire des images non seulement sur des matériaux dégénérés, mais sur des matériaux non dopés et semi-isolants. Nous donnerons des exemples d'observation sur des substrats de GaAs massifs, sur des structures épitaxiales de GaAs et des composés pseudo-binaires.

2. Conditions d'observation des images. - 2.1 RÉSOLUTION. - La résolution des images dépend du diamètre $\mathrm{du}$ faisceau électronique au niveau de l'échantillon, de celui de la poire de diffusion des électrons primaires créés dans le matériau et enfin de la longueur de diffusion des porteurs minoritaires qui détermine le volume final d'où provient l'émission lumineuse.

\subsubsection{Dimensions du spot. - Dans les conditions}

normales d'utilisation du microscope électronique à balayage, c'est-à-dire en électrons secondaires ou rétrodiffusés, le diamètre du spot n'excède généralement pas une centaine d'angströms. Cette valeur est très faible devant les autres limitations de la résolution que nous analyserons dans la suite. Cependant dans le cas de l'utilisation en cathodoluminescence, il est nécessaire d'augmenter le débit du faisceau, ce qui conduit à une augmentation de diamètre du spot électronique. $\mathrm{Ce}$ diamètre dépend également de la divergence du faisceau au niveau de l'échantillon, de la brillance du filament ainsi que de l'aberration de sphéricité des lentilles (dans nos conditions de travail, les aberrations chromatiques et de diffraction sont négligeables). Pour connaître la dimension du spot il faut donc tracer le réseau de courbes donnant cette dimension en fonction de ces paramètres. La figure 1 donne le résultat de ces calculs effectués d'après le modèle de Thornton [14] et les données du constructeur pour notre appareil (JEOL JSM U 3). Les courants maximaux utilisés seront de 2 à $3 \times 10^{-7} \mathrm{~A}$ à $20 \mathrm{kV}$. Les courbes de la figure 1 montrent qu'avec ces courants la dimension peut être conservée très inférieure au micron à condition d'utiliser une divergence suffisamment élevée, c'est-à-dire un diaphragme large et une distance de travail faible.

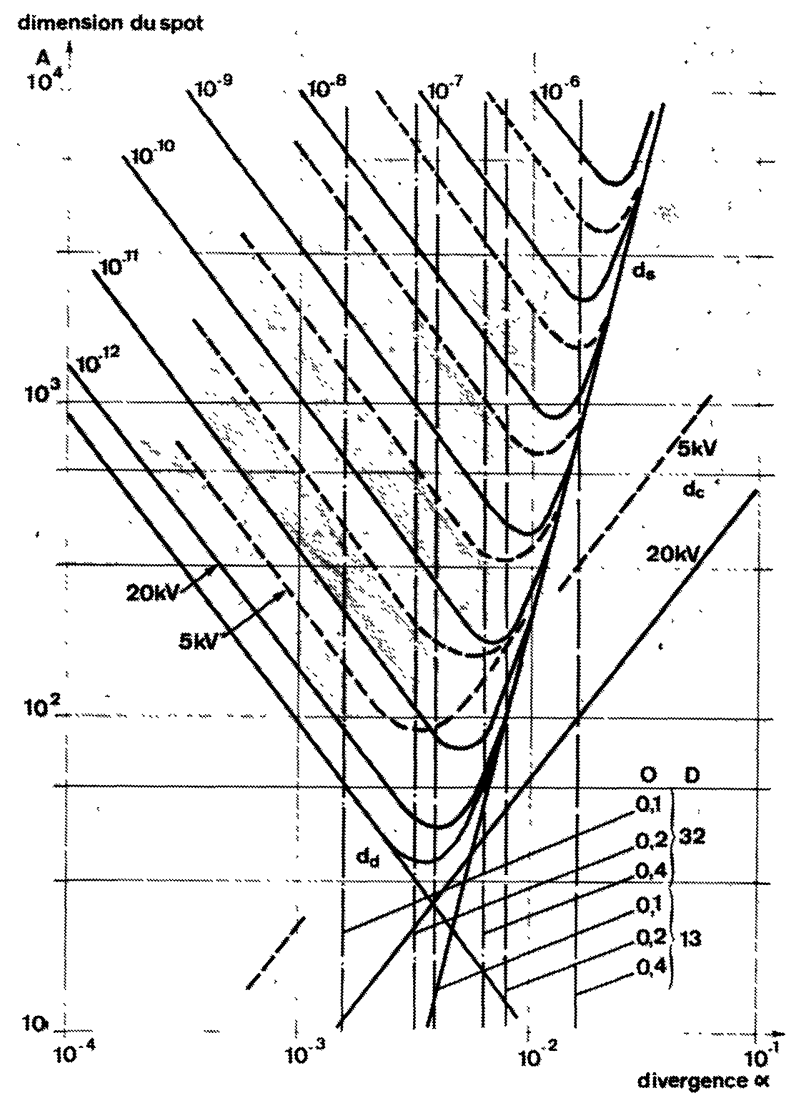

FIG. 1. - Dimensions du spot (en angstroems) en fonction de la divergence du faisceau ( $\alpha$ en radian) et du courant échantillon. Courbe continue $E_{0}=20 \mathrm{keV}$. Courbe en tirets $E_{0}=5 \mathrm{keV}$. Les lignes verticales correspondent à 6 différentes conditions expérimentales. $\varnothing$ diamètre du diaphragme et $D$ distance échantillon-diaphragme. 
2.1.2 Rayon de la poire d'interaction. - Le modèle le plus simple et le plus couramment utilisé pour rendre compte de la pénétration des électrons dans un solide consiste à supposer une décroissance exponentielle d'énergie le long de chaque rayon vecteur d'une sphère centrée à une profondeur $X_{\mathrm{e}}$ [19], le paramètre de l'exponentielle est donné par $\mu=8,8 \rho 10^{2} E_{0}^{-1,65}$ avec $\mu$ en $\left(\mathrm{cm}^{-1}\right), \rho$ la densité du matériau en $\left(\mathrm{mg} / \mathrm{cm}^{3}\right)$ et $E_{0}$ l'énergie des électrons incidents en $(\mathrm{keV})$. On définit un rayon pratique pour la sphère soit $\left(R_{\mathrm{B}}-X_{\mathrm{e}}\right)$ avec $R_{\mathrm{B}}=11 / \mu\left(R_{\mathrm{B}}\right.$ est le " range de Bethe »). A titre d'exemple, pour GaAs, avec $E_{0}=20 \mathrm{keV}$, on obtient $R_{\mathrm{B}} \simeq 3,3 \mu \mathrm{m}$ d'autre part $X_{\mathrm{e}}=4 R_{\mathrm{B}} /(Z+4)$ [13], $Z$ est le numéro atomique de l'élément, soit pour GaAs à $20 \mathrm{keV} X_{\mathrm{e}} \simeq 0,4 \mu \mathrm{m}$.

Il faut noter que le rayon $\left(R_{\mathrm{B}}-X_{\mathrm{e}}\right)$ de la poire d'excitation n'a pas de limites bien définies, ces calculs ne fournissant donc qu'un ordre de grandeur qui ne permet pas de tirer une conclusion pratique. Expérimentalement nous avons essayé de déterminer le profil de diffusion des électrons dans les solides à partir de l'étude des électrons rétrodiffusés sur un coin d'échantillon. La figure 2 montre le schéma de
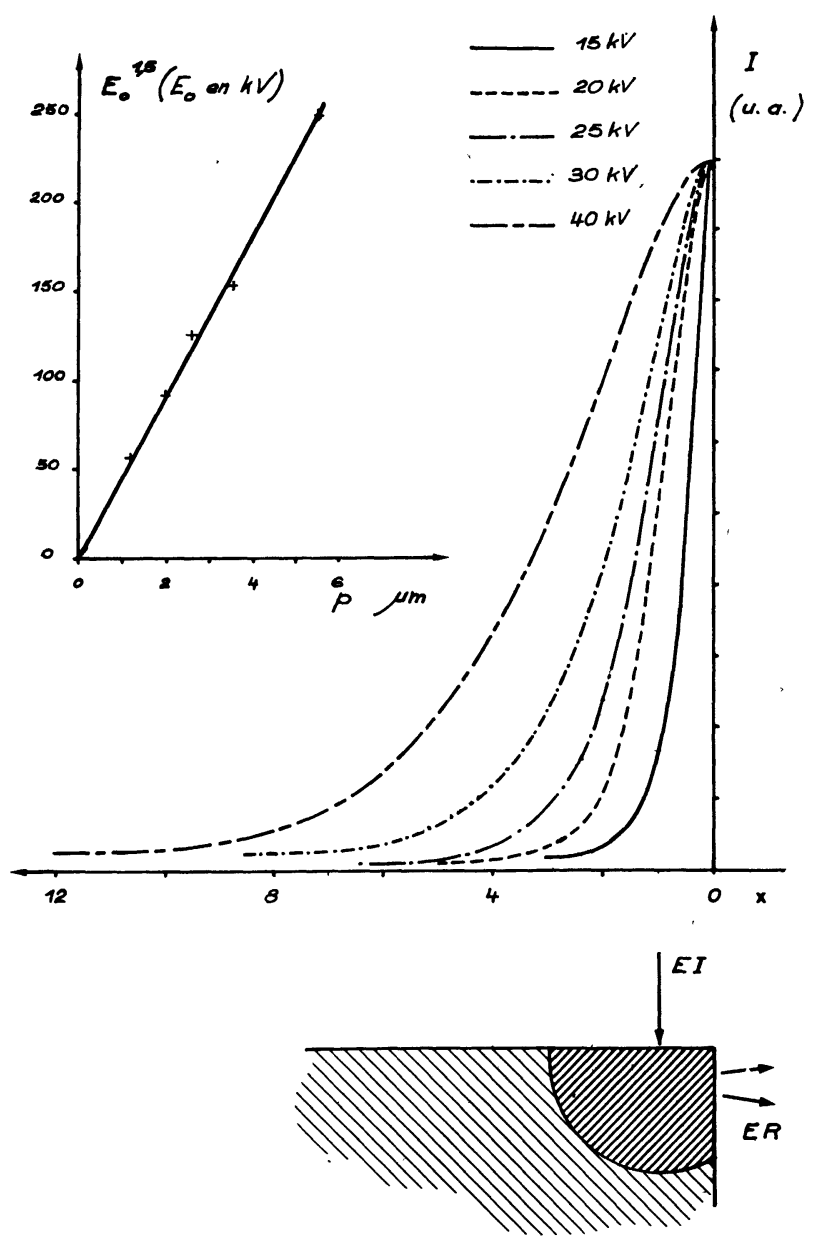

FIG. 2. - Etude de la rétrodiffusion sur un bord d'échantillon clivé. Profils $I(x)$ obtenus à différentes tensions d'accélération. $x$ est la distance du spot au bord de l'échantillon. La partie encadrée donne la variation de $P$ avec la tension d'accélération $E_{0}$. principe de l'expérience et les profils obtenus pour $E_{0}$ sont de $15 \mathrm{kV}$ à $40 \mathrm{kV}$. L'encadré montre que $(P)$ varie pratiquement comme $E_{0}^{1.5}$. Cette expérience constitue un moyen de vérification particulièrement simple du modèle d'interaction de Klein. On peut alors prendre comme résolution le double de la largeur à mi-hauteur des profils, ce qui fournit à $20 \mathrm{kV}$ environ $2 \mu \mathrm{m}$.

2.1.3 Longueur de diffusion. - Outre la limitation de résolution provenant des dimensions de la poire d'interaction, il faut tenir compte de la diffusion des paires électrons-trous qui agrandit la zone d'où proviennent les photons. En fait, comme dans toute expérience de luminescence, ce sont les porteurs minoritaires qui interviennent et c'est donc leur longueur de diffusion qui altère la résolution des images. Pour préciser ce phénomène, il faut résoudre l'équation de diffusion des porteurs avec une fonction de génération en volume qui est déterminée par la perte d'énergie différentielle des électrons primaires. Cette résolution ne peut être mise à son terme que dans une seule dimension, par exemple la profondeur dans le matériau, mais les phénomènes en profondeur donnent une assez bonne idée des autres directions du fait que la diffusion est supposée sphérique. Les résultats montrent qu'avec une longueur de diffusion de $1 \mu \mathrm{m}$ et à $20 \mathrm{kV}$ la résolution passe de $2 \mu \mathrm{m}$ à $2,5 \mu \mathrm{m}$. Donc la résolution paraît être peu affectée pour les longueurs de diffusion inférieures au micron, ce qui est le cas des matériaux fortement dopés ou semi-isolants. Par contre, dans les matériaux purs, la longueur de diffusion en l'absence d'injection peut atteindre quelques microns [20] mais nous verrons que dans ces matériaux, l'injection devient importante et par conséquent limite la longueur de diffusion.

En conclusion, la résolution des images de cathodoluminescence sera de $2 \mu \mathrm{m}$ environ à $20 \mathrm{keV}$ pour la plupart des matériaux.

2.2 Collection de la lumière. Auto-absorption. - Les semiconducteurs ayant des indices de réfraction élevés (pour GaAs $n=3,4$ ), l'angle maximum pour lequel les photons émis sortent effectivement de l'échantillon est faible: environ $18^{\circ}$ pour GaAs. Ce phénomène, outre qu'il diminue considérablement l'intensité de cathodoluminescence, provoque une importante anisotropie de l'émission dans l'espace avec un maximum dans la direction normale à la surface. Nous avons représenté sur la figure 3 la disposition géométrique à l'intérieur du microscope ; même en position basse de l'échantillon, il n'est pas possible de recueillir la lumière sous incidence normale, l'angle maximum admissible est de $70^{\circ}$ ce qui conduit à un angle d'incidence des électrons de $50^{\circ}$. Cependant il faut remarquer qu'aux très faibles angles, la collection de la lumière est favorisée par les réflexions parasites à l'intérieur du microscope.

Pour de nombreuses expériences, en particulier lorsqu'il s'agit de clivages, on est amené à étudier 


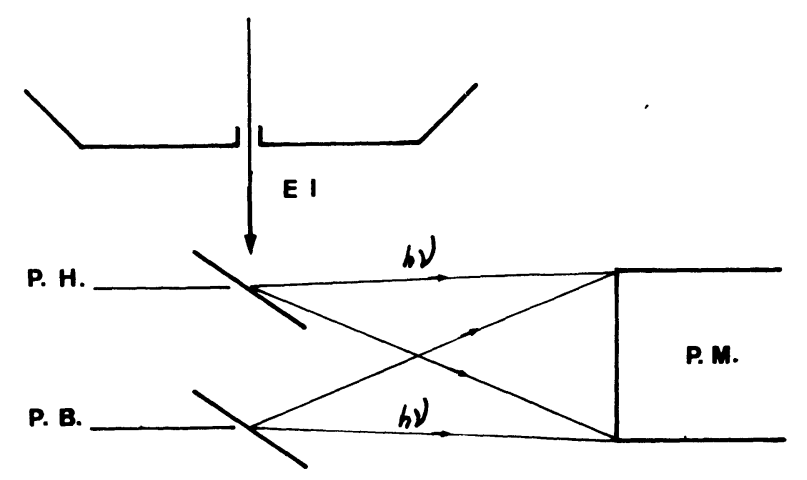

FIG. 3. - Schéma illustrant la collection de luminescence pour les deux distances de travail : $D=13 \mathrm{~mm}$ et $D=32 \mathrm{~mm}$.

des bords d'échantillons. Dans ces conditions, il faut tenir compte de la sortie de la lumière par les deux faces de l'échantillon.' Cet effet modifie sensiblement les images de cathodoluminescence comme le montre la figure 4 . Il s'agit de l'étude d'un coin sur
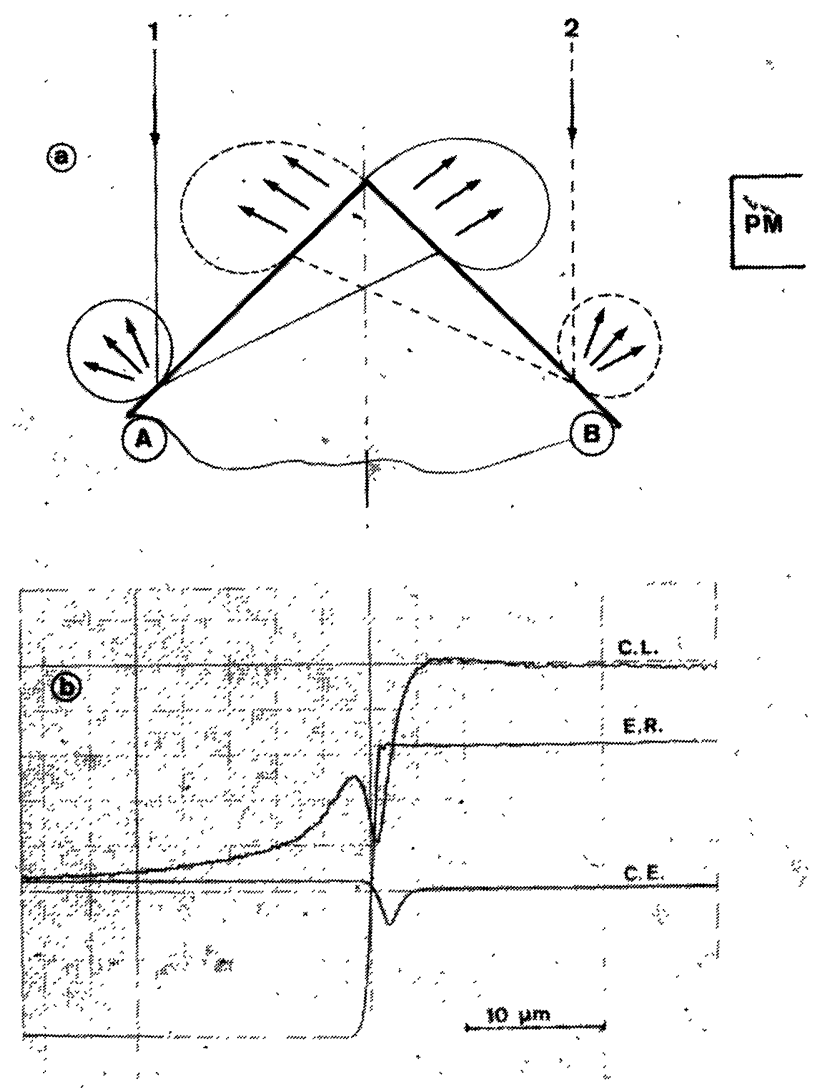

Fig. 4. - Etude de la collection optique sur un coin pour un échantillon de $\mathrm{GaAs} n+\mathrm{Si}-10^{18}$.

Fig. 4a. - Disposition géométrique de l'expérience : on a figuré les faisceaux lumineux sortant de l'échantillon pour deux positions du faisceau d'électrons 1 et 2 .

FIG. 4b. - Profils de cathodoluminescence CL, courant échantillon CE et électrons rétrodiffusés ER.

du GaAs massif homogène dont on a clivé une face. On observe lorsque le faisceau atteint la face cachée $\mathbf{A}$ une luminescence qui croît très fortement près du bord de l'échantillon. Le phénomène inverse se produit pour la face $B$. Ce profil s'explique si on considère que pour chaque position du spot deux faisceaux lumineux sortent de l'échantillon, le faisceau normal à la surface et le faisceau parallèle à la surface qui atteint la face opposée avec un angle inférieur à $18^{\circ}$. $\mathrm{Ce}$ dernier faisceau doit parcourir une distance importante avant de sortir du matériau, par conséquent les phénomènes d'auto-absorption doivent être pris en considération. Ils compliquent la variation du signal lorsqu'on se rapproche du bord. Nous avons vérifié que cette courbe est bien exponentielle comme on peut le prévoir pour' un tel phénomène, nous en avons déduit le coefficient d'absorption de la lumière émise soit $\sim 3 \times 10^{3} \mathrm{~cm}^{-1}$. Le coefficient est relativement faible devant celui correspondant aux transitions bande à bande $\left(\simeq 10^{4} \mathrm{~cm}^{-1}\right)$ ce qui montre que la cathodoluminescence provient ici d'une émission mettant en jeu les queues de bandes. Notons que le signal faible obtenu lorsqu'on se déplace sur $\mathrm{A}$ loin $\mathrm{du}$ bord provient de réflexions parasites. L'interprétation du profil suivant $B$ est strictement identique en inversant le rôle des deux faisceaux. Enfin le creux observé au niveau du clivage correspond à la diminution du courant échantillon dans cette zone provoquée par l'augmentation de la rétrodiffusion (cf. 2.2). Nous avons vérifié ce point expérimentalement en enregistrant le profil du courant échantillon.

Ce type de phénomène, outre qu'il permet d'accéder au coefficient d'absorption de la luminescence qui donne une indication sur la largeur d'onde d'émission, ne doit pas être négligé chaque fois que l'on étudie des clivages.

2.3 EChauffement de LA zone IRRAdiéE. - Pour un faisceau de $20 \mathrm{kV}$ et de $10^{-7} \mathrm{~A}$, la puissance sur l'échantillon est d'environ $200 \mathrm{~kW} / \mathrm{cm}^{2}$. Nous avons calculé l'échauffement induit dans l'échantillon en se plaçant dans un modèle de diffusion hémisphérique. Dans les conditions définies ci-dessus pour GaAs on trouve que l'élévation de température maximale au point d'impact est de $15^{\circ} \mathrm{C}$ et tombe à $10^{\circ} \mathrm{C}$ à $1 \mu \mathrm{m}$ de profondeur. Cet échauffement n'est donc pas trop important à la température ambiante, par contre il devient non négligeable lorsqu'on travaille à basse température. Pour $\mathrm{CdTe}$, les mêmes calculs donnent des échauffements 10 fois plus élevés dont il faudra tenir compte pour interpréter les résultats.

2.4 ETUDE DE L'INTENSITÉ DE LA CATHODOLUMINESCENCE. - L'intensité de luminescence dépend du nombre de paires électron-trou créées dans le matériau et de leur probabilité de recombinaison radiative. Le nombre de paires électron-trou créées est directement proportionnel au nombre d'électrons incidents. Ces paires électron-trou peuvent ensuite se recombiner soit par transition radiative en donnant naissance à un photon soit par des transitions non radiatives ou par des recombinaisons en surface. 
2.4.1 Calcul de l'injection. - Le nombre de paires créées par seconde est

$$
g=\frac{i E_{0}}{e e_{i}}
$$

où $E_{0}$ et $i$ sont respectivement l'énergie et le débit d'électrons, $e_{i}$ est l'énergie de création d'une paire (environ 4,4 eV pour GaAs [19]), et $e$ la charge de l'électron. Le nombre de porteurs en excès est $\delta N=g \tau$ où $\tau$ est la durée de vie totale des porteurs et la densité en excès est $\Delta n=g \tau / V$ où $V$ est le volume dans lequel se répartissent ces paires, c'est-à-dire, si on se reporte aux courbes de la figure 2, environ $50 \mu \mathrm{m}^{3}$ (on a supposé que la répartition s'effectue dans une demi-sphère de $3 \mu \mathrm{m}$ de rayon). Théoriquement on obtient alors :

$$
\Delta n=5 \times 10^{32} i \tau \quad i \text { en } \mathrm{A}, \tau \text { en } \mathrm{s} .
$$

A titre d'exemple, dans $\operatorname{GaAs}(n)$ la durée de vie totale des trous est d'environ $10^{-8} \mathrm{~s}$, pour un courant de $10^{-7} \mathrm{~A}$, l'injection sera de $\Delta n=5 \times 10^{17} \mathrm{~cm}^{-3}$. On constate que cette valeur est importante et que par conséquent on doit en tenir compte dans la détermination de la durée de vie des porteurs.

2.4.2 Calcul de l'intensité de cathodoluminescence. - Cette intensité peut s'écrire :

$$
I=\alpha \eta g
$$

où $\eta$ est le rendement quantique interne et $\alpha$ le rendement de collection des photons qui est constant. Si $\tau_{\mathrm{r}}$ et $\tau_{\mathrm{nr}}$ sont les durées de vie radiative et non radiative dans le matériau, on a

$$
\eta=\frac{1 / \tau_{\mathrm{r}}}{1 / \tau_{\mathrm{nr}}} \text { avec } \frac{1}{\tau}=\frac{1}{\tau_{\mathrm{r}}}+\frac{1}{\tau_{\mathrm{nr}}}
$$

dans le cas d'expériences effectuées à basse injection, ce rapport est constant, mais dans nos conditions il variera avec l'injection puisque $\tau_{\mathrm{r}}$ pour des transitions bande à bande dépend du nombre de porteurs libres soit

$$
\frac{1}{\tau_{\mathrm{r}}}=\beta n
$$

durée de vie des $t^{+}$( $\beta$ est une constante du matériau), le nombre d'électrons libres $n$ s'écrit :

$$
n=n_{0}+\Delta n
$$

où $n_{0}$ est le nombire de porteurs à l'équilibre et $\Delta n$ le nombre d'électrons en excès qui dépend de $\tau$ par l'intermédiaire de (2) donc

$$
\frac{1}{\tau_{\mathrm{r}}}=\beta\left(n_{0}+\Delta n\right)
$$

soit

$$
\Delta n=5 \times 10^{32} i \frac{\tau_{\mathrm{nr}}\left[\beta\left(n_{0}+\Delta n\right)\right]^{-1}}{\tau_{\mathrm{nr}}+\left[\beta\left(n_{0}+\Delta n\right)\right]^{-1}}
$$

$\Delta n$ est alors solution d'une équation du second degré

$$
\frac{\beta}{5 \times 10^{32} i} \Delta n^{2}+\frac{1}{5 \times 10^{32} i}\left[\frac{1}{\tau_{\mathrm{nr}}}+\beta n_{0}\right] \Delta n-1=0
$$

ce qui fournit l'intensité de luminescence

$$
I=\alpha \frac{\beta\left(n_{0}+\Delta n\right)}{\frac{1}{\tau_{\mathrm{nr}}}+\beta\left(n_{0}+\Delta n\right)} g
$$

$g$ étant donné par (1).

Différents cas doivent être envisagés :

1) Matériaux peu dopés en forte injection $\left(\Delta n \gg n_{0}\right)$ dans lesquels la durée de vie non radiative est faible devant la durée de vie radiative soit

$$
\frac{1}{\tau_{\mathrm{nr}}} \gg \beta\left(n_{0}+\Delta n\right)
$$

l'équation se simplifie et on a $\Delta n \simeq 5 \times 10^{32} i \tau_{\mathrm{nr}}$ qui fournit

$$
I=5 \times 10^{32} \alpha \beta \tau_{\mathrm{nr}}^{2} i^{2} \frac{E_{0}}{e e_{i}}
$$

$I$ varie comme $i^{2}$.

2) Matériaux peu dopés en injection faible à durée de vie radiative faible $\Delta n \ll n_{0}$; on obtient

$$
\Delta n=5 \times 10^{32} i \tau_{\mathrm{nr}}
$$

et

$$
I=\beta n_{0} \tau_{\mathrm{nr}} i \frac{E_{0}}{e e_{i}}
$$

3) Matériaux peu dopés mais à forte durée de vie non radiative; il peut se faire que l'injection et la constante $\beta$ soient telles que

$$
\beta n_{0} \ll \frac{1}{\tau_{\mathrm{nr}}} \ll \beta \Delta n .
$$

Dans ces conditions $\Delta n$ varie comme $i^{1 / 2}$ mais $I$ varie encore linéairement.

4) Matériaux fortement dopés, on a toujours $n_{0} \gg \Delta n$ d'où

$$
I=\frac{\alpha \beta n_{0}}{\frac{1}{\tau_{\mathrm{nr}}}+\beta n_{0}} i \frac{E_{0}}{e e_{i}}
$$

et varie donc toujours linéairement avec $i$ puisque le terme devant $g$ ne dépend pas de $i$.

2.4.3 Etude expérimentale de l'influence de l'injection. - D'après les formules précédentes donnant l'intensité de cathodoluminescence, on constate que suivant la nature de l'échantillon les comportements doivent être très différents.

Nous avons porté sur la figure 5 les courbes $\log I=f(\log i)$ pour une série d'échantillons de 


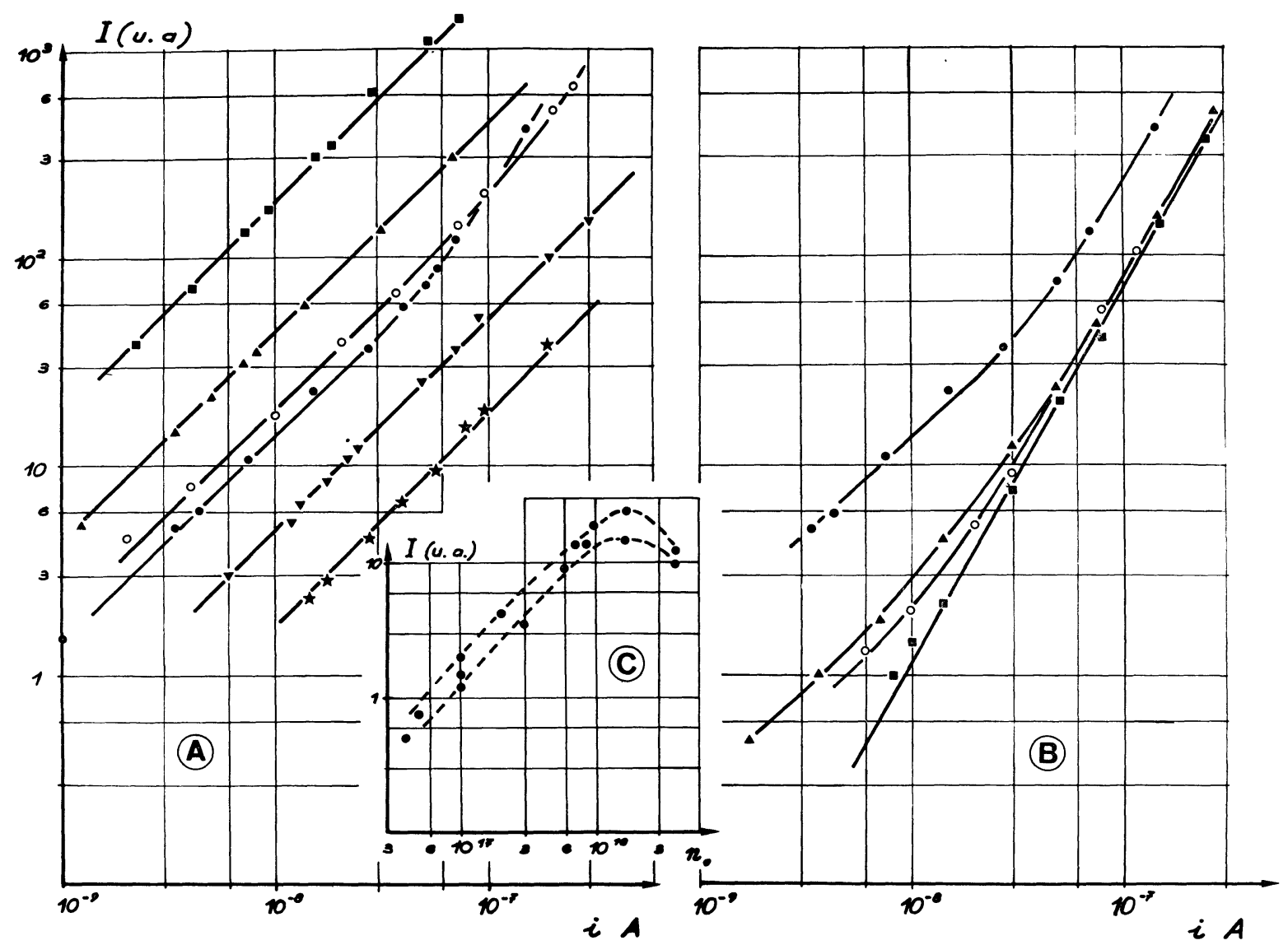

Fig. 5. - Influence de l'injection sur l'intensité dẹ luminescence $\left(E_{0}=20 \mathrm{kV}\right)$.

Fig. 5a. - Matériaux fortement dopés : $10^{18}, \boldsymbol{\Delta} 2 \times 10^{17}, \bigcirc 10^{17}, \nabla 10^{16}, * 4 \times 10^{18}$;

Fig. 5b. - Matériaux peu dopés : 1017, $\triangle 10^{16}, \bigcirc 2 \times 10^{15}$, $10^{14}$.

Fig. 5c. - Variation de l'intensité de luminescence en fonction du niveau de dopage à intensité de faisceau fixe.

type $n$, massifs ou épitaxiques. Ces résultats ont été obtenus pour tous les échantillons dans les mêmes conditions de collection optique et d'amplification du signal, le spot électronique étant maintenu à sa valeur minimale pour chaque courant (Fig. 1). On vérifie bien que pour les dopages supérieuŕs à $10^{18} \mathrm{~cm}^{-3}$, $I$ varie toujours linéairement avec $i$, alors que pour les dopages plus faibles la situation se complique. Par exemple, l'échantillon massif RT 580 dopé à $10^{16} \mathrm{~cm}^{-3}$ reste linéaire ce qui montre que l'injection est dans ce cas inférieure à $10^{16} \mathrm{~cm}^{-3}$, la durée de vie des porteurs minoritaires dans ce matériau est donc faible.

Par contre l'échantillon X 130 dopé à $2 \times 10^{14}$ (épitaxique) suit une loi purement quadratique; les autres échantillons présentent des situations intermédiaires : le XI 102 montre clairement l'existence de deux régions, la séparation entre les deux correspond à $\Delta n \simeq n_{0}$, il est possible d'en déduire la durée de vie du matériau grâce à (2) : on trouve

$$
\tau=7 \times 10^{-9} \mathrm{~s} .
$$

Cette étude nous permettra de discuter l'origine des contrastes dans les divers types de matériaux.
2.4.4 Influence des recombinaisons en surface. On peut rendre compte de l'influence de la surface en introduisant dans l'équation de diffusion une vitesse de recombinaison en surface $s$. En reprenant la fonction de génération, il est possible de tracer les profils de l'injection $\Delta n(X)$ pour différentes valeurs de $s(*)$. La valeur couramment admise pour $s / D$, $D$ étant le coefficient de diffusion $\left(D=L^{2} / \tau\right)$ est $s / D \simeq 10^{5} \mathrm{~cm}^{-1}$ [6] ; numériquement on observe que cette valeur conduit à des résultats voisins de ceux obtenus à $s=\infty$. On note en outre que pour ces valeurs de recombinaison, la cathodoluminescence provient principalement d'une zone utile s'étendant à $20 \mathrm{kV}$ sur $2 \mu \mathrm{m}$ en dessous de la surface, avec une région moins active près de celle-ci.

3. Observation des défauts dans les matériaux très dopés. - La planche 1 représente une série d'images de cathodoluminescence obtenues sur des échantillons de $\operatorname{GaAs}(n)$ massifs dopés au tellure. On voit apparaître différents types de défauts :

1) Des dislocations qui se présentent sous la forme de lignes plus ou moins longues $\left(\mathrm{D}_{2}\right.$ par exemple)

(*) Humbert, A., Communication privée. 

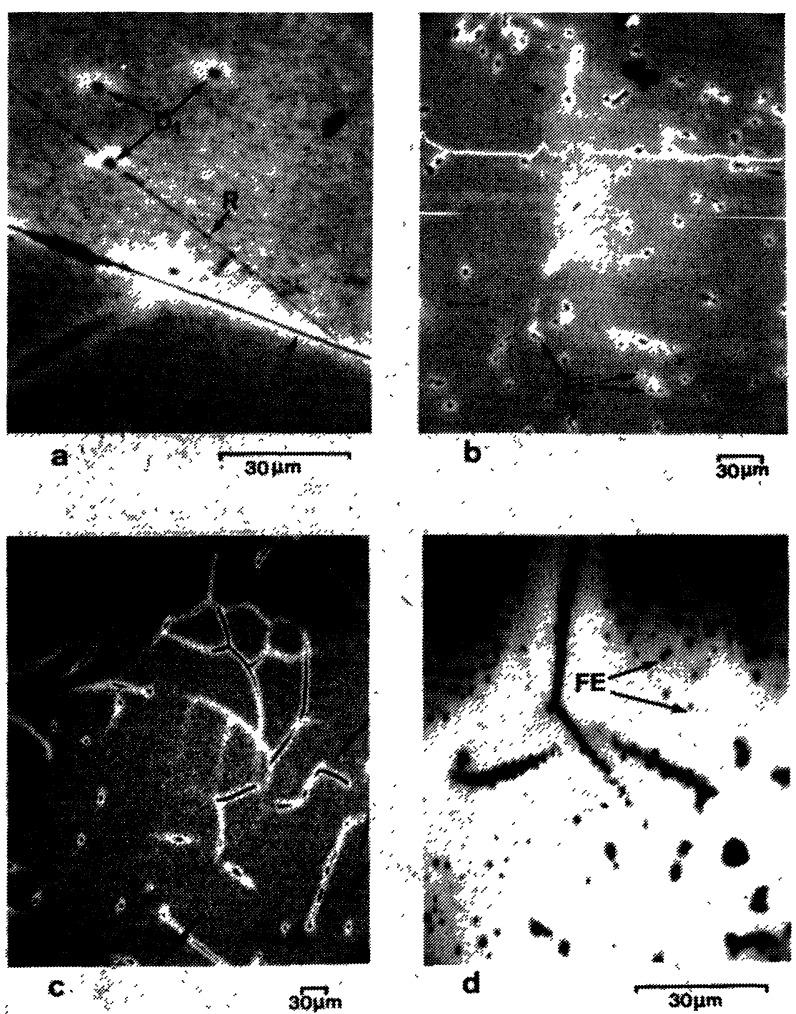

Planche 1. - Images de luminescence de substrats de GaAs $n+$ Te $n>10^{18} \mathrm{~cm}^{-3}$. a) Image d'un clivage (partie basse) et surface (partie haute) $\mathrm{R}$ rayure, $\mathrm{C}$ coin de l'échantillon, $D_{1}$ dislocations émergeant à la surface, $D_{2}$ dislocations émergeant à la surface et sur le clivage. b) Image de dislocations et profil sur une ligne. FE faute d'empilement. c) Réseau de dislocations parallèles à la surface et mise en évidence des zones de purification du matériau autour des dislocations. $d$ ) Précipitations, fautes d'empilement (FE) et dislocations décorées.

ou de pointes d'émergence $\left(D_{1}\right.$ par exemple). Nous avons vérifié par des décapages ioniques que ces défauts étaient bien du type linéaire dans les deux cas. La figure $a$ représente une image obtenue sur un coin d'échantillon. La partie supérieure de l'image correspond à la surface de l'échantillon et la partie inférieure à la face de clivage. Le bord de ce clivage est matérialisé par la ligne C (cf. Fig. 4), en outre on note la présence d'une rayure de polissage $\mathrm{R}$. La dislocation $\mathrm{D}_{2}$ (Fig. 4) présente un phénomène intéressant puisqu'elle émerge sur les deux faces du coin. On peut donc approximativement déterminer sa position par rapport à lui, et en déduire la profondeur à partir de laquelle la dislocation devient visible au-dessous de la surface; on trouve environ $2 \mu$ ce qui est en bon accord avec les estimations faites paragraphe 2 à $20 \mathrm{kV}$ qui est la tension utilisée pour cette figure.

2) Des précipités visibles sur $c$ et $d$. Comme il s'agit là de matériaux dopés au tellure, ce sont probablement des précipités de tellure ou d'un de ses composés. En outre remarquọns que beaucoup de ces points sombres apparaissant sur $b$ et $d$ sont appairés et il est vraisemblable qu'il s'agit plutôt de fautes d'empilement que de précipités. De telles fautes ont été observées dans GaAs par microscopie électronique en transmission (*). La photo $1 b$ montre un échantillon ne contenant pas de précipités dans les zones homogènes.

3) Des différences importantes de la luminescence d'un point à l'autre du cristal qui sont corrélées avec la présence des dislocations.

Comme nous l'avons vu en 2.4 , dans les matériaux très dopés du type de ceux étudiés sur la planche 1 , l'intensité de la cathodoluminescence dépend $\mathrm{du}$ dopage $n_{0}$ [21] et de la durée de vie non radiative $\tau_{\mathrm{nr}}$. Nous avons porté sur la figure $5 c$ l'intensité de cathodoluminescence en fonction du dopage pour des conditions constantes du faisceau d'électrons. On observe que pour $n_{0}<10^{18}$, cette intensité croît avec $i$, sensiblement linéairement ce qui explique, d'après la formule (7), que $1 / \tau_{\mathrm{nr}} \gg \beta n_{0}$. Par contre, au-delà de cette valeur, l'intensité de la cathodolu. minescence décroît, traduisant ainsi une diminution de la durée de vie non radiative. Ce phénomène provient de la précipitation du dopant au-dessus de $10^{18} \mathrm{~cm}^{-3}$. Tous les matériaux de la planche 1 étaient dopés à plus de $10^{18} \mathrm{~cm}^{-3}$; il est possible d'expliquer le contraste autour des dislocations en considérant que le tellure diffuse autour des dislocations et vient précipiter sur celles-ci. Les zones entourant alors les lignes de dislocations sont plus faiblement dopées et le rendement de cathodoluminescence augmente conformément à ce qui apparaît dans la figure $5 c$. La photo $b$ montre le type de profil obtenu sur une ligne de balayage coupant une dislocation; on observe que les variations du signal ne sont pas aussi intenses que le contraste des images le laisse penser. La variation du taux de dopage est d'environ $20 \%$ et l'étendue de la zone purifiée atteint $20 \mu \mathrm{m}$. La photo $1 d$ montre, à l'appui de cette interprétation, que lẹs dislocations sont décorées de précipités ce qui, en outre, explique qu'elles soient matérialisées par une ligne sombre. Dans certaines zones sur la photo $c$ il apparaît des dislocations pour lesquelles on n'observe pas cette ligne sombre alors que la région purifiée, plus fortement luminescente traduit leur existence. Ce cas correspond à des dislocations qui sont pratiquement parallèles à la surface mais situées trop en profondeur pour qu'elles apparaissent sur l'image autrement que par leur zone d'interaction. Le même phénomène peut provenir des dislocations situées au-dessus de la surface qui donc ont été éliminées par décapage.

La dimension apparente des taches noires indiquant la présence de précipités est de l'ordre du micron, mais cela ne signifie pas qu'il s'agisse là de la dimension des précipités puisque la zone entourant un précipité doit être perturbée sur une distance beaucoup plus grande que les dimensions du précipité. Deux effets peuvent être considérés : celui des contraintes et celui de la perturbation électrostatique des

(*) Dupuy, M., Communication privée. 
bandes par alignement du niveau de Fermi du semiconducteur avec celui du précipité métallique.

Nous avons porté sur la planche 2 des images prises sur du $\operatorname{GaAs}(n)$ dopé au silicium; elles mon-

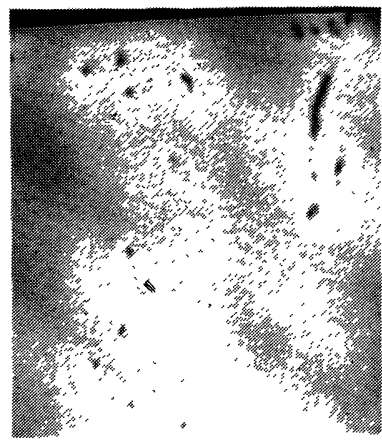

a

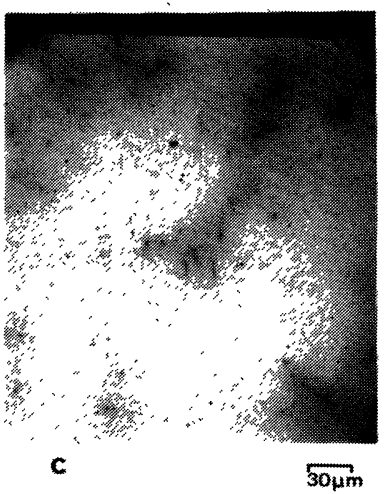

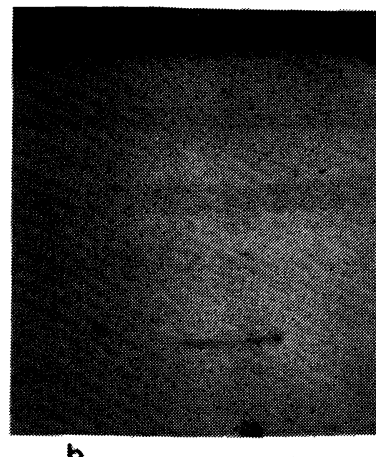

b

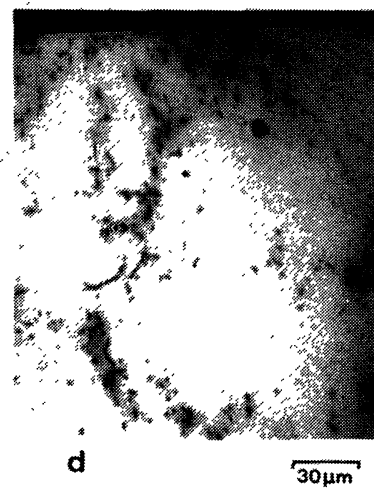

Planche 2. - Evolution du contraste autour des dislocations dans des substrats de GaAs $n+\mathrm{Si}: a) n=3 \times 10^{17} ; b$ ) et c) $n=10^{18}$; d) $n=2 \times 10^{18}$; absence de précipitations.

trent que le silicium ne précipite pas comme le tellure, du moins jusqu'à $2 \times 10^{18}$ qui est la limite des dopages étudiés. De plus, les contrastes entourant la dislocation sont inversés. Dans l'échantillon $a$ qui est dopé à $3 \times 10^{17}$ on observe des zones claires entre des dislocations, ce qui montre que le dopage de ces régions a augmenté (par le tellure il diminuait). On pourrait penser que l'augmentation de la luminescence se traduit par une variation du dopage $n_{0}$ ou une augmentation de $\tau_{\mathrm{nr}}$. Cependant les échantillons $b, c$ et $d$ présentent des contrastes différents : pratiquement nul pour $b$ (dopé à $10^{18}$ ), et sombre autour des dislocations pour $d$ (dopé à $2 \times 10^{18}$ ). Cette observation s'explique en considérant que dans le cas de $b$ on est au maximum de la courbe $I\left(n_{0}\right)$ (cf. Fig. 5c) alors que pour $d$ on est dans la zone décroissante, d'où l'inversion des contrastes par rapport à $a$. Des variations de $\tau_{\mathrm{nr}}$ conduiraient à des effets identiques dans tous les échantillons. Finalement on constate que le silicium est attiré par les dislocations et forme une atmosphère autour de celle-ci sans qu'il y ait précipitation.

La planche 3 représente en $a$ et $b$ les images d'une couche épitaxiale de $\operatorname{GaAsP}(n)$. Sur le clivage $a$

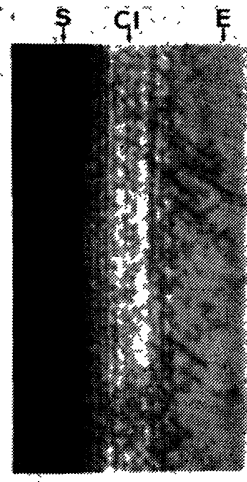

a

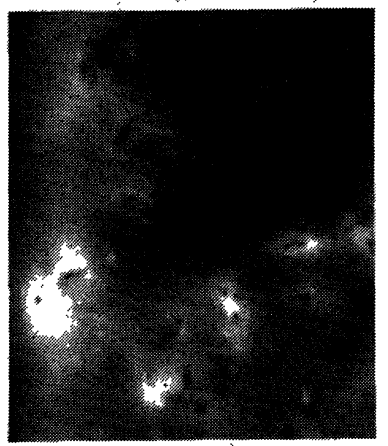

c

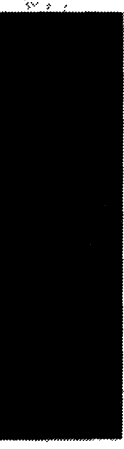

$20 \mu \mathrm{m}$

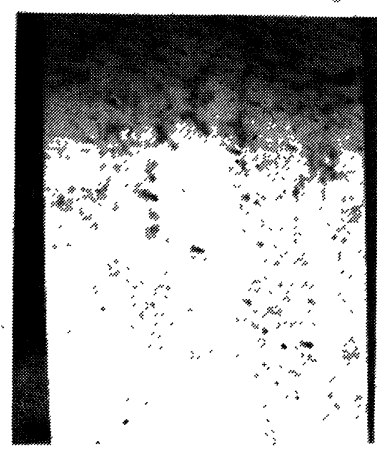

$20 \mathrm{~mm}$

$20 \mu \mathrm{m}$ b

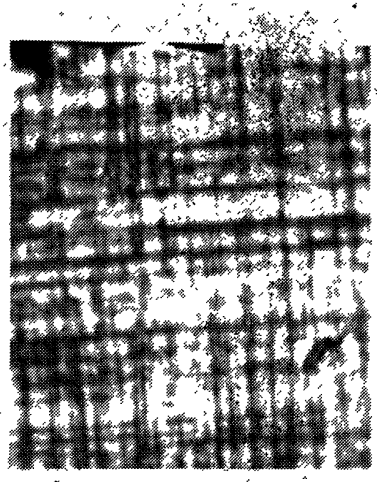

$20 \mu \mathrm{m}$

Planche 3. - a) Clivage d'une couche épitaxiale de composés ternaires GaAsP. $\mathbf{S}=$ substrat, $\mathrm{CI}=$ couches intermédiaires, $\mathrm{E}=$ couche finale ; $b$ ) Image dans le plan de dépôt des dislocations de rattrapage ; $c$ ) Image effectuée en régime quadratique sur un matériau massif peu dopé ; $d$ ) Idem à $c$ ), mais sur matériau épitaxique.

on observe les couches intermédiaires de rattrapage du paramètre entre le substrat (GaAs) et la couche finale dans laquelle subsiste un certain nombre de dislocations qui se sont propagées depuis la zone intermédiaire. La photo $b$ montre l'image obtenue parallèlement à la surface de la plaquette après décapage chimique dans la zone intermédiaire. On y observe clairement le réseau des dislocations de rattrapage du paramètre. Compte tenu de la tension d'accélération $(12 \mathrm{kV})$ et de la profondeur de visualisation telle que nous l'avons discutée en II $(\sim 1 \mu \mathrm{m})$ on peut calculer la densité de dislocations parallèles à la surface soit $7 \times 10^{7} \mathrm{~cm}^{-2}$. Cette valeur est en bon accord avec les prévisions théoriques [22].

4. Observation des défauts dans les matériaux peu dopés. - La planche 3 montre également des images de matériaux peu dopés à très forte injection (zone quadratique). On observe encore les lignes de dislocations entourées de zones claires sur la photo $c$ qui correspond à un matériau massif, alors que ce type de phénomène ne se produit pas sur les matériaux épitaxiques $d$. Puisque l'on est en régime quadratique dans les deux cas, l'intensité de cathodoluminescence suit une loi du type (6), elle ne dépend donc que de $\tau_{\mathrm{nr}}$. La zone claire autour des dislocations sur $c$ 
traduit donc une augmentation de cette durée de vie non radiative par la disparition de défauts non radiatifs dans la zone d'interaction de la dislocation. Ce phénomène ne se produit pas dans les échantillons obtenus par épitaxie. Il a été observé précédemment que la présence de dislocations dans des échantillons de GaAs massifs du type de ceux utilisés ici provoque une diminution de l'absorption optique moyenne dans le domaine du proche infrarouge (autour de $1,2 \mathrm{eV}$ ) [23]. Cette absorption provient vraisemblablement de défauts intrinsèques tels que des lacunes de gallium [24]. Les défauts non radiatifs, dont la diminution provoque l'augmentation de la luminescence autour des dislocations sont probablement les mêmes que ceux qui sont observés en absorption. Les images de cathodoluminescence démontrent alors bien l'effet des dislocations sur l'absorption.

Il apparaît donc que les dislocations provoquent une diminution de la densité des lacunes sur une distance d'environ $20 \mu \mathrm{m}$ de la même manière qu'elles induisent des inhomogénéités de dopage.

Enfin, sur les figures $3 c$ et $3 d$, les dislocations ellesmêmes apparaissent toujours comme des lignes ou des points sombres, ce qui indique que même en l'absence de précipitation du dopant, les dislocations jouent le rôle de centres non radiatifs. On peut en donner deux interprétations. D'une part, elles peuvent concentrer autour d'elles une grande densité de défauts non radiatifs, d'autre part, elles ont un effet électrostatique et de contrainte sur les bandes d'énergie du solide [25].

5. Etude des structures épitaxiales. - Nous avons utilisé les observations de cathodoluminescence pour étudier la répartition des défauts dus aux structures épitaxiales de GaAs sur GaAs. Les substrats étaient soit du týpe $\mathrm{n}^{+}$soit semi-isolants. Nous avons essayé de déterminer les conditions de formation et d'élimination des défauts depuis l'interface substrat-épitaxie jusqu'à la couche finale. Les observations ont été réalisées sur des clivages d'échantillon ou sur des biseaux afin d'étendre l'image, et sur la surface des plaquettes par décapages successifs.

La planche 4 (photo $a$ ) montre l'image de clivage d'une épitaxie $\mathrm{n}$ (qq $10^{15} \mathrm{~cm}^{-3}$ ) sur un substrat dopé $\mathrm{Si}\left(\mathrm{qq} 10^{18} \mathrm{~cm}^{-3}\right.$ ) à faible injection. Dans ces conditions, la luminescence du substrat et de l'épitaxie est faible. On retrouve cependant les dislocations dans le substrat. Le phénomène intéressant est l'apparition au niveau de l'interface, côté substrat, d'une couche fortement luminescente sur $30 \mu$ environ. Cette zone se retrouve sur le profil de cathodoluminescence tracé pour une épitaxie $\mathrm{n}^{+} \mathrm{nn}^{+}$portée dans la planche $5 a$. Pour déterminer l'origine de ce phénomène nous avons réalisé des spectres de photoluminescence à $4 \mathrm{~K}$ de ces mêmes matériaux après divers décapages. On note que dans la zone concernée il apparaît, outre les raies d'émission habituelles du $\mathrm{GaAs}$, des raies provenant de la présence du cuivre [26].

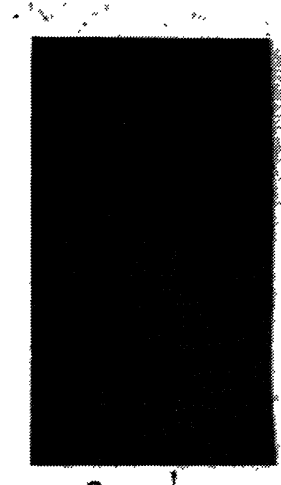

a. $\stackrel{s}{s} n^{*}$
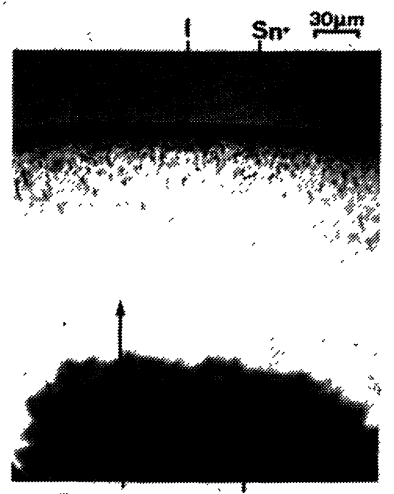

C. En

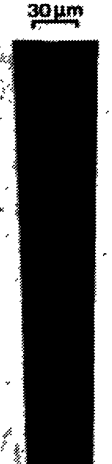

$\mathrm{bE}$

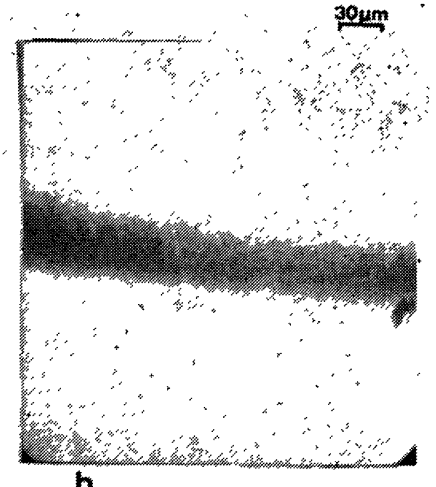

b.

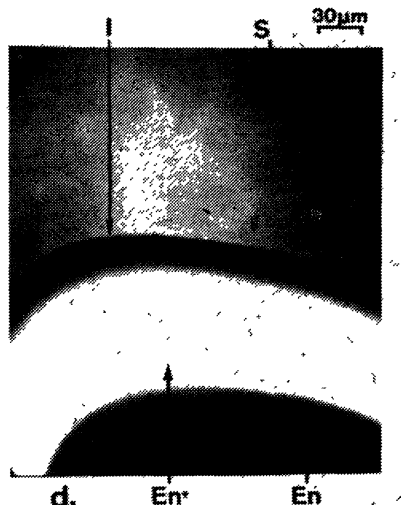

d.
Planche 4. - Applications aux structures épitaxiales GaAs/ GaAs. a) Couche épitaxiale avec diffusion de cuivre $\mathrm{Sn}+$ substrat, $\mathrm{Zd}=$ zone diffusée, $\mathrm{E}=$ couche épitaxiale ; $b$ ) Détail de la zone interfaciale I non radiative de la figure $d ; c$ ) et $d$ ) résultats obtenus sur biseau de couches épitaxiales $S, n^{+}, n$, $\mathrm{Sn}^{+}$substrat, I interface, $\mathrm{En}^{+}$épitaxie $\mathrm{n}^{+}$, En épitaxie $\mathrm{n}$.

Il semble donc que la zone luminescente provienne d'une diffusion du cuivre pendant la croissance épitaxique. D'ailleurs il est actuellement possible d'éliminer totalement la présence du cuivre dans les réacteurs d'épitaxie, ce qui conduit à la disparition du phénomène comme le montre le profil de la figure $b$ (planche 5). L'augmentation de la luminescence peut provenir de l'émission propre des niveaux associés au cuivre (entre 1,3 et $1,35 \mathrm{eV}$ ) ou d'un effet secondaire sur la durée de vie des porteurs.

Sur les profils $a$ et $b$ (planche 5) il apparaît aussi, indépendamment de la présence du cuivre, une zone étroite faiblement luminescente au niveau de l'interface. Elle traduit la grande densité de défauts existant à l'interface. Nous avons analysé cette région en photoluminescence mais aucune émission particulière n'a pu y être détectée ce qui prouve qu'il ne s'agit pas d'un phénomène de diffusion des impuretés ou d'introduction de lacunes. Les photos $4 c$ et $4 d$ montrent les images obtenues sur des biseaux pour deux épitaxies $\mathrm{n}^{+} \mathrm{n}$ sur substrat $\mathrm{n}^{+}$. Sur la photo $c$, on observe une grande densité de dislocations qui prennent naissance à l'interface. Sur $d$, il n'y a pas de dislocations d'interface par contre la chute de luminescence existe toujours et un agrandissement 

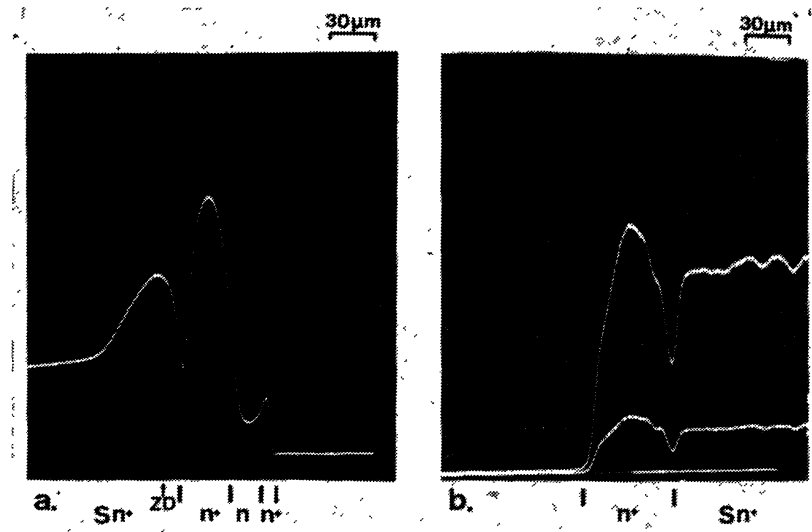

soum
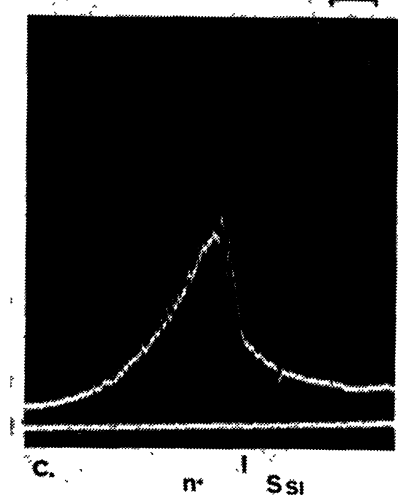

$n^{*}$ ss

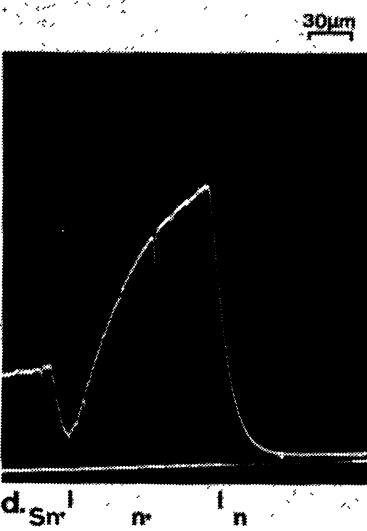

Planche 5. - Profils de cathodoluminescence : a) Clivage d'une épitaxie $\mathrm{n}^{+}, \mathrm{n}, \mathrm{n}$ avec diffusion de cuivre (ZD) ; b) Clivage d'une épitaxie $\mathrm{n}^{+}, \mathrm{n}$ sans diffusion de cuivre ; $c$ ) Biseau d'une épitaxie $\mathrm{n}^{+}>4 \times 10^{18} \mathrm{~cm}^{-3}$ sur substrat semi-isolant ; $d$ ) Biseau d'une épitaxie $n^{+} 10^{18}$ sur substrat $n^{+}$.

de cette zone (photo 4b) montre qu'elle contient de très nombreux microprécipités (de l'ordre de $10^{11} \mathrm{~cm}^{-3}$ ). Cette précipitation provoque une diminution du dopage et par conséquent explique la chute de cathodoluminescence puisque la zone épitaxique $\mathrm{n}^{+}$est dopée à $\mathrm{qq} 10^{17} \mathrm{~cm}^{-3}$. La photo $5 d$ montre le profil de cathodoluminescence obtenu. On observe que les défauts s'éliminent progressivement à mesure que l'on s'éloigne de l'interface ; à $10 \mu$, l'élimination n'est pas totale puisque le signal de cathodoluminescence n'est pas encore stabilisé. Sur la photo $5 c$, nous avons porté le cas d'une étude identique (épitaxie $\mathrm{n}^{+}$) mais à un niveau de dopage de $4 \times 10^{18} \mathrm{~cm}^{-3}$; on se trouve alors dans la zone décroissante de $I\left(n_{0}\right)$ (cf. Fig. $5 c$ ) et le phénomène s'inverse : la cathodoluminescence diminue lorsqu'on s'éloigne de l'interface, ce qui traduit bien toujours une augmentation du dopage.

Ces observations ont été utilisées pour déterminer l'épaisseur minimale nécessaire des couches tampons dans les épitaxies vapeur $\mathrm{n}^{+} \mathrm{nn}^{\neq}$et également pour l'optimisation des débuts de croissance.

6. Conclusions. - Les images de cathodoluminescence obtenues au MEB sur Ga^s montrent trois sortes de contrastes reliés à des variations de concentration du dopant, à des variations locales de la durée de vie non radiatives dues aux défauts du cristal et enfin à des zones non semiconductrices telles que les précipités. Nous avons montré que les matériaux fortement dopés présentent des contrastes de la $1^{\text {re }}$ et $3^{\mathrm{e}}$ catégorie, alors que les variations de durée de vie apparaissent dans les matériaux peu dopés à condition que l'injection soit suffisante. Ce taux d'injection peut être estimé si on connaît la durée de vie et la longueur de diffusion des porteurs minoritaires dans un matériau, de même la résolution des images de cathodoluminescence dépend directement de la longueur de diffusion.

Le MEB apparaît alors comme un moyen souple, de mise en œuvre entièrement simple, pour étudier les défauts dans les cristaux semiconducteurs, pourvu qu'ils soient luminescents.

Nous avons ainsi étudié la précipitation du dopant dans des cristaux de GaAs fortement dopés et démontré que si le tellure et le soufre souvent utilisés comme dopant $\mathrm{n}$ précipitent au-dessus de $10^{18} \mathrm{~cm}^{-3}$, le silicium ne précipite pas.

Les images de cathodoluminescence ont également permis de mettre en évidence les effets des dislocations dans ces cristaux. Dans les matériaux dopés au tellure on observe un important phénomène de pompage du tellure par les dislocations, il conduit à la formation de précipités sur les dislocations et d'une zone à dopage plus faible sur qq $10 \mu \mathrm{m}$ autour d'elles. Par contre dans le cas du dopage au silicium, il y a création de régions plus fortement dopées autour des dislocations. Dans les matériaux non dopés, les dislocations induisent une amélioration du rendement radiatif sur des distances équivalentes; nous avons émis l'hypothèse que cet effet traduit l'introduction des dislocations et des lacunes.

Enfin, nous avons étudié les phénomènes se produisant à l'interface d'une croissance épitaxique de GaAs sur GaAs. Trois types de défauts ont été mis en évidence : les diffusions du cuivre lorsqu'il est présent dans le substrat, la formation des dislocations à l'interface et la précipitation du dopant. Ces défauts concernent plusieurs dizaines de microns autour de l'interface. Du point de vue pratique, cette observation a conduit à réaliser des couches tampons en début de croissance.

Des phénomènes identiques ont été relevés dans des cristaux de CdTe ou de ZnTe.

Dans l'avenir il sera possible d'améliorer la résolution de ces analyses en refroidissant les échantillons ce qui diminue la longueur de diffusion et augmente dans des proportions importantes le rendement radiatif des matériaux. De plus, l'installation d'un système de sélection des longueurs d'onde doit permettre de mieux comprendre certaines images. 


\section{Bibliographie}

[1] Pankove, J. I., J. Phys. Soc. Japan 21 (1966) 298.

[2] Rakshit, S. et al., Phys. Stat. Sol. 4 (1971) 249.

[3] Rakshit, S., Bisuuas, S. N., Chakravarti, A. N., $J$. Phys. C 5 (1972) 702.

[4] Casey, H. C., Kaiser, R. H., J. Electrochem. Soc., Solid State Science 114 (1967) 149.

[5] Alferov, Zh. I. et al., Soviet Phys. Semicond. 3 (1970) 1234.

[6] Pinard, P., Thèse, Lyon 1964.

[7] Wittry, D. B., Kyser, D. F., J. Appl. Phys. 38 (1967) 375.

[8] Rao-Sahib, T. S., Wittry, D. B., J. Appl. Phys. 40 (1969) 3745.

[9] Thornton, P. R., IEEE Trans. Electron Devices ED 16 (1969) 360.

[10] Czaja, W., J. Appl. Phys. 37 (1966) 4236.

[11] McAvoy, B. R. et al., Appl. Phys. Lett. 14 (1969) 16.

[12] Wittry, D. B., Appl. Phys. Lett. 8 (1966) 142.

[13] Wittry, D. B., Kyser, D. F., J. Appl. Phys. 35 (1964) 2439.
[14] Thornton, F. R., Scanning Electron Microscopy. (Chapman sons) 1968.

[15] Cazabat, M., Schiller, C., Diguet, D., Lefevre, J. C., Colloque AVISEM, Versailles (sept. 1971).

[16] Schiller, C., Colloque caractérisation, Grenoble 1972, à paraître.

[17] Schiller, C., 4th Conference on GaAs and Related Compounds, Boulder (sept. 1972).

[18] Hallais, J., Humbert, A., Schiller, C., Colloque annuel GFCC, Clermont-Ferrand (1973).

[19] Klein, C. A., Appl. Optics 5 (1966) 1922.

[20] Hwang, C. J., J. Appl. Phys. 42 (1971) 4408.

[21] Cusano, D. A., Solid State Commun. 2 (1964) 353.

[22] Abrahams, M. S., Weiberg, L. R., Buiocchi, C. J., Blanc, J., J. Mater. Sci. 4223, 1969 b.

[23] Leyral, P., Pinard, P., Bois, D., J. Solid State Chem. 6 (1973) 406.

[24] Bois, D., Pinard, P., Phys. Stat. Sol. (a) 7 (1971) 85.

[25] Benoît a La Guillaume, C., Thèse, Paris 1958.

[26] Farre, E., Phys. Stat. Sol. (a) 9 (1972) 259. 